\title{
Towards temporal cognition for robots: a neurodynamics approach
}

\author{
Weronika Wojtak* $^{* \dagger}$, Flora Ferreira*, Luís Louro*, Estela Bicho* and Wolfram Erlhagen ${ }^{\dagger \S}$ \\ * Algoritmi Center, University of Minho, Portugal \\ $\dagger$ Center of Mathematics, University of Minho, Portugal \\ §Email: wolfram.erlhagen@math.uminho.pt
}

\begin{abstract}
If we want robots to engage effectively with humans in service applications or in collaborative work scenarios they have be endowed with the capacity to perceive the passage of time and control the timing of their actions. Here we report result of a robotics experiment in which we test a computational model of action timing based on processing principles of neurodynamics. A key assumption is that elapsed time is encoded in the consistent buildup of persistent population activity representing the memory of sensory or motor events. The stored information can be recalled using a ramp-to-threshold dynamics to guide actions in time. For the experiment we adopt an assembly paradigm from our previous work on natural human-robot interactions. The robot first watches a human executing a sequence of assembly steps. Subsequently, it has to execute the steps from memory in the correct order and in synchrony with an external timing signal. We show that the robot is able to efficiently adapt its motor timing and to store this information in memory using the temporal mismatch between the neural processing of the sensory feedback about executed actions and the external cue.
\end{abstract}

\section{INTRODUCTION}

Adequate behavior in a dynamic environment requires the ability to detect and exploit its temporal regularities. In almost all of our daily activities, we make use of these regularities to determine when relevant events will occur and how to time our actions towards them. Consider for instance a joint action task like setting the dinner table in which we coordinate our actions with those of another person. Sensing the flow of time is important to monitor and predict the behavior of our partner and to select a complementary action at an appropriate time. Based on previous experiences with the collaborative task, we are reasonably accurate in predicting how long our partner takes to pick up a plate and set it on the table. This in turn allows us to time the placing of a napkin on top of the plate in order to achieve a tight temporal coordination in service of fluent task execution. Moreover, we are able to rapidly adjust our temporal prediction about the partner's action and the timing of our own action if unexpected delays are explained by environmental cues, e.g., the plates have to be picked up from a different location than usual.

In current robotics research, the question how to endow autonomous robots with the capacity to learn and exploit temporal regularities of the environment is to a large extent unexplored ([17], for example studies [3], [5], [11]). This is perhaps not surprising giving that in highly controlled industrial settings the precise timing of robot motion can be pre-specified by the designer and controlled by a computer clock. As robots move into human populated environments it will become increasingly important for them to achieve a human-like temporal prediction and adaptation capacity. Indeed, a recent user study of human-robot interaction in an object transfer task reveals that the temporal precision of the robot action has a greater weight for the subjective experience rating than the spatial precision [14].

A promising design approach towards robots with a temporal cognition capacity is to take inspiration how the brain represents temporal information [15]. One hypothesis is that time is inherent in the neural dynamics of cell assemblies that are engaged in other cognitive computations [10]. For instance, the short-term memory (STM) of a briefly presented cue is thought to be stored in the persistent activity of neural populations in higher brain areas. Elapsed time since cue onset can be robustly encoded in the consistent increase of population activity during a delay period [13], [16]. We have recently implemented the notion of shared neural resources for STM and time measurement in a dynamic neural field (DNF) model of sequence learning [9], [22]. The information about the order and relative timing of successive stimulus events is stored in an activation gradient of persistent activity over stimulus-specific subpopulations. The information can be recalled in a decision field by integrating this pattern as subthreshold input and applying a ramp-to-threshold dynamics to all subpopulations.

The present study set out to investigate in a real-world robotics experiment the plausibility of an extension of our neurodynamics model that addresses the challenge of adaptive motor timing in relation to perceived temporal features of the environment. Concretely, we adopt the assembly paradigm used in our previous research on natural human-robot interaction [2], [19]. The robot first watches a human realizing a sequence of assembly steps. Subsequently, the robot has to recall the sequence from memory while planning its action timing in order to synchronize the realization of each assembly step with the occurrence of an external cue.

The rest of the paper is structured as follows: we start with a brief presentation of basic neural processing principles implemented in DNF models, followed by a description of the model architecture and its functionalities (section 2). We then present the experimental paradigm and specify assumption we made (section 3). In Section 4, we describe the results of the robotics experiment. The paper finishes with a general 
discussion of concepts and results (section 5).

\section{DYNAMIC FIELD MODEL}

\section{A. Model description}

Dynamic Neural Fields (DNFs) represent a theoretical framework for developing cognitive robot control architectures that is consistent with fundamental principles of cortical information processing in distributed networks of connected neural populations [7]. Task-relevant information is represented by supra-threshold activity patterns (or bumps) of local populations. These patterns are self-sustained in the absence of any external drive due to recurrent excitatory and inhibitory interactions within the populations. To implement a memory function, a stable bump state must coexist with a homogeneous resting state such that the memory can be switched on and off by sufficiently strong transient input from sensors and/or connected populations. Weaker inputs lead to a subthreshold preshaping of neural populations which nevertheless may have important consequences for the computations in a distributed network (see below, [7]). Since no changes in synaptic weights are involved, the processing mechanisms explained by DNF theory support a fast, activation-based type of learning [19]. Figure 1 presents an overview of the model architecture with several interconnected dynamic neural fields. The Perceptual Memory field $u_{S M}$ stores a sequence of stimulus events as a multi-bump pattern. The strength of each memory representation reflects the time elapsed since stimulus presentation resulting in an activation gradient from the first to the last event (for details see [9]). The two bottom layers show the model extension for adaptive motor timing in sequence recall. The neural computations in the Action Onset Memory field $u_{A M E M}$ implement a motor-related memory pattern of the sequence. The strength of individual bumps controls the timing of action onset during recall in order to finish the individual actions in synchrony with an external cue. Starting with the perceptual memory pattern which $u_{A M E M}$ receives as excitatory input, the bump amplitudes are adjusted according to a detected temporal mismatch between the time courses of suprathreshold activity in the Cue field $u_{S}$ and the Feedback field $u_{F}$, representing the external synchronization signal and the sensory feedback about executed actions, respectively [22]. Both fields receive in addition to the sensory input also the pattern of $u_{A M E M}$ as subthreshold excitation. The action onset field $u_{A C T}$ is also preshaped by the motor memory meaning that the first event in the motor sequence has the highest and the last event the lowest preactivation. Sequence recall starts with a continuous increase of the baseline activation level due to the release of proactive inhibition [21]. This causes a ramplike buildup of activity of all subpopulations until a threshold associated with action onset is reached. To monitor progress and avoid repetition errors, bumps in the Past Events field $u_{P E}$, which evolve in response to excitatory input from $u_{F}$, cause through inhibitory connections a suppression of existing excitations at corresponding sites of the three fields in the recall layer.

\section{B. Model equations}

The population dynamics in each field is governed by the one-dimensional model proposed by Amari [1]:

$\tau \dot{u}(x, t)=-u(x, t)+S(x, t)+\int w(x-y) f(u(y, t)) \mathrm{d} y-h(x, t)$,

where $u(x, t)$ represents the activity at time $t$ of the neuron at location $x, \tau$ defines the time scale of the field dynamics which is adapted to the scale of the robotics experiments, and $S(x, t)$ represents the time dependent, localized input at site $x$ from connected populations and/or the sensory system (vision, proprioception). The resting state, which may depend on time and space, is defined by $h(x, t)<0$. The nonlinear transfer function $f(u)$ is taken as the Heaviside step function with threshold 0 . The intra-field couplings $w(x, y)=w|x-y|$ between neurons are assumed to depend on distance only. To ensure the stability of multiple bumps in the fields with a memory function, we use an oscillatory coupling function with multiple zero crossings (for details see [8]):

$$
w(x)=A^{-b|x|}(\operatorname{bin}|\alpha x|+\cos (\alpha x)),
$$

with $A>0, b>0$ and $b<\alpha<1$. In the fields of the recall layer, in which only a single bump should exist at any time, we adopt a kernel for which inhibition dominates at larger distances:

$$
w(x-y)=w_{e x c} e^{\left((x-y)^{2} / 2 \sigma_{e x c}^{2}\right)}-w_{i n h i b},
$$

with $w_{\text {exc }}>0, \sigma_{\text {exc }}>0$ and $w_{\text {inhib }}>0$.

The strength of individual memory representations in $u_{S M}$ is controlled by a state-dependent dynamics of the resting activity $h_{S M}(x, t)[6]:$

$$
\begin{aligned}
\dot{h}_{S M}(x, t)= & \left(1-f\left(u_{S M}(x, t)\right)\right)\left(-h_{S M}(x, t)+h_{S M_{0}}\right) \\
& +\frac{1}{\tau_{h_{S M}}} f\left(u_{S M}(x, t)\right),
\end{aligned}
$$

where $f(u)$ represents again the Heaviside step function, $h_{S M_{0}}<0$ defines the level to which $h_{S M}$ converges without suprathreshold activity at position $x$, and $\tau_{h_{S M}}>0$ measures the growth rate when it is present. This dynamics implements a linear increase of supra-threshold activity with time. To retrieve the order and timing information stored in the activation gradient of $u_{A C T}$, we apply a linear dynamics for the inhibitory input $h_{A C T}<0$ defining the resting state:

$$
\tau_{h_{A C T}} \dot{h}_{A C T}(t)=1, \quad h_{A C T}\left(t_{0}\right)=h_{A C T_{0}}<0 .
$$

Note that by changing the value of $\tau_{h_{A C T}}$ the sequence can be recalled with different speed, while preserving the relative timing of motor events.

The adaptation of bump amplitudes in $u_{A M E M}$ to represent action timing is performed by applying the following $h$ dynamics:

$$
\begin{aligned}
\dot{h}_{A M E M}(x, t)= & \beta\left(1-f\left(u_{S}(x, t)\right) f\left(u_{F}(x, t)\right)\right) \\
& \left(f\left(u_{F}(x, t)\right)-f\left(u_{S}(x, t)\right)\right),
\end{aligned}
$$




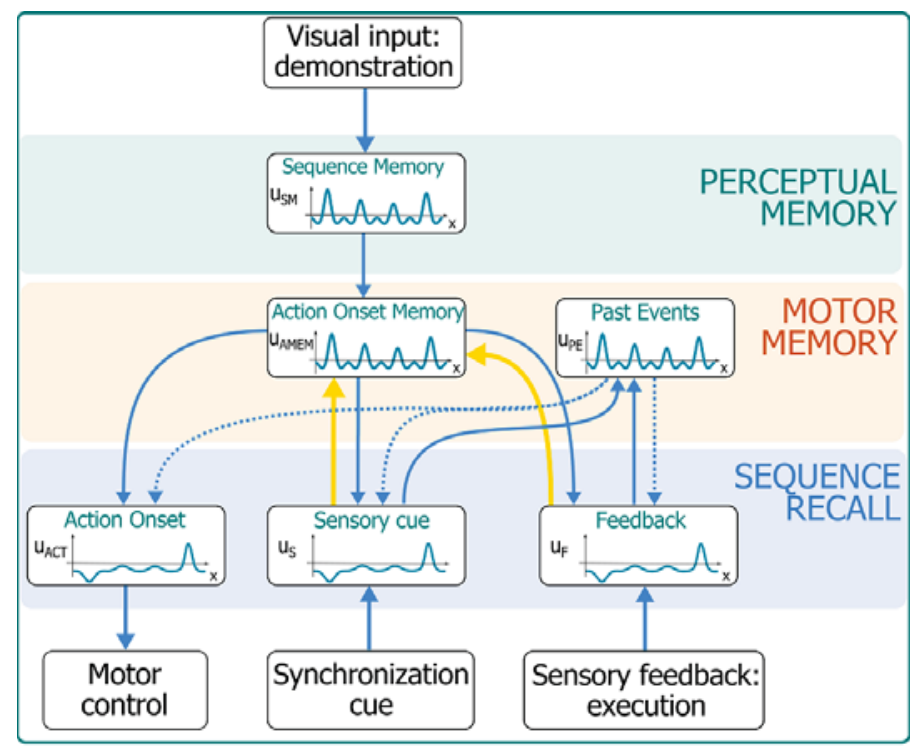

Anara Neural field

$\Lambda$ Neural population

$\longrightarrow$ Excitatory connection

.......... Inhibitory connection Adaptation of activity gradient in $\mathrm{u}_{\text {AMEM }}$

Fig. 1: Schematic view of the DNF architecture with several interconnected fields implementing perceptual sequence memory, motor sequence memory and sequence recall.

where $f(u)$ is again the Heaviside step function and $\beta>0$ is the adaptation rate parameter. Typically, the evolution of activity in $u_{F}$ will be initially delayed compared to $u_{S}$. The adaptation dynamics will thus cause an increase of bump amplitude to initiate action onset earlier in the next trial.

\section{TASK DESCRIPTION}

For the experiments we used ARoS, the humanoid robot developed by our group [2]. It consists of a stationary torus on which a seven degrees of freedom arm with a three-fingered hand and a stereo camera system with a pan-tilt unit are mounted. To test the adaptive motor timing capacity in a real-world experiment, we adopted a subsequence of a toy assembly paradigm that we have used in our previous research on natural human-robot interactions [2], [19]. ARoS has to grasp three parts from a table and attach them in a specific order to a base: insert left wheel (ILW), insert left nut (ILN) and insert column 1 (IC1) (see Fig. 2). While watching a human performing the sequence, ARoS first memorizes the order and the relative timing of individual assembly steps. Fig. 3 (left) shows the camera view of the wheel already attached and the hand still on the wheel which we define as the moment in time when the subgoal has been achieved. The visual recognition of the scene integrates knowledge about position, shape and color of objects, hand tracking is facilitated using a color marker [19]. Subsequently, ARoS executes the sequence from memory with an additional timing constraint for the achievement of each assembly step defined by a blue synchronization cue displayed on a screen (Fig. 3, right). This shall mimic the common situation in joint action that identical

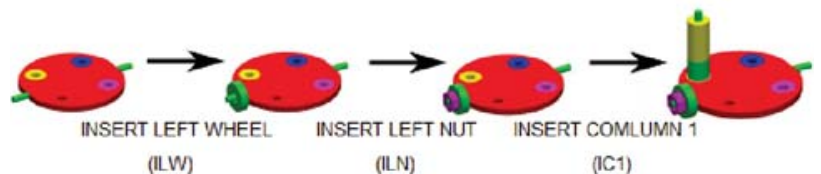

Fig. 2: An overview of the task structure.
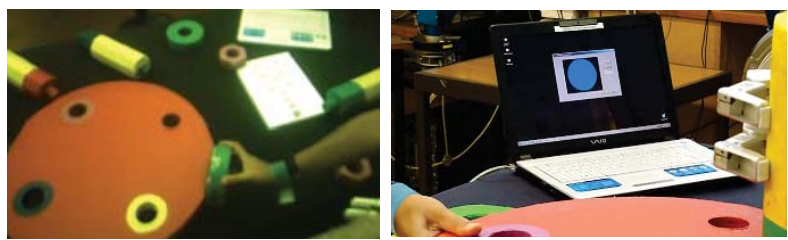

Fig. 3: Robot camera views showing the achievement of the first assembly step (left) and the synchronization cue (right).

actions (e.g., assembly of the two wheels) or complementary behaviors (e.g., fluent object transfer) should be achieved in synchrony by the co-workers. Note that since the color cue is not subtask specific, we apply a constant input to all neurons in the preshaped Cue field $u_{S}$ at the time of cue presentation. The start and the end of the sequence are signaled by cues with different colors, green and red, respectively. The time between the green cue and the blue cue thus defines the permitted execution time of each step, and ARoS has to learn to time its movements in order to meet this constraint. While vision could be used as well, we use for simplicity proprioceptive signals at the time when ARoS starts to release the object as input to $u_{F}$ and to measure the level of synchrony.

\section{RESUlts}

In the following we present the results of the robotics experiments with the focus on the adaptive timing capacity (for a video of the whole experiment see http://marl.dei.uminho.pt/public/videos/icdl.html). Fig. 4 shows snapshots of the video sequence (left column) and the execution phase (right column) together with the corresponding states of the population activities in the perceptual memory field $u_{S M}$ and the Action Onset field $u_{A C T}$, respectively. As can be seen in the bottom row on the left, ARoS has memorized the achievement of all assembly steps and their relative timing as shown by the three bumps with decreasing amplitude. The snapshot of $u_{A C T}$ on the right shows a suppression of the neural populations representing the first two steps below resting state, meaning that they have been already executed. The bump at position IC1 indicates that the robot should have already started the manipulation of the column which is in line with its behavior as shown by the video snapshot.

The temporal evolution of bumps in $u_{S M}$ in response to the localized input from the vision system about the executed assembly steps is shown in Fig. 5. The bump amplitude increases linearly with elapsed time meaning that the relative timing of perceptual events is stored in the amplitude difference. The increase stops with the presentation of the red 


\begin{tabular}{|l|c|c|c|}
\hline & Action 1 & Action 2 & Action 3 \\
\hline Recall 1 & 26 & 32 & 45 \\
Recall 2 & 2 & 1 & 1 \\
\hline
\end{tabular}

TABLE I: Delay (in seconds) during task execution before (top) and after (bottom) adaptation of movement onset time.

cue indicating the end of the sequence (not shown).

At the beginning of the first execution trial, the Action Onset Memory $u_{A M E M}$ equals the pattern in $u_{S M}$ memorized during the demonstration. Consequently, also the level of pre-activation of each subpopulation in $u_{A C T}$ already reflects serial order of task execution. When the h-dynamics in $u_{A C T}$ is triggered by the green cue, all sub-populations will reach sequentially the threshold for movement initiation. However, since movement duration is not yet reflected in the memory representation, ARoS will finish each assembly step with a significant delay compared to the synchronization cue. This can be seen by comparing the time courses of activation in the three fields of the recall layer Fig. 7(a). Moreover, the delays are different for each assembly step reflecting different movement and manipulation durations. Fig. 6 compares the stored activation gradient in $u_{S M}$ (solid line) with the pattern in $u_{A M E M}$ (dashed line) after the application of the adaptation rule which compares the time courses of activation in the Cue field $u_{S}$ and the Feedback field $u_{F}$ individually for all subpopulations. The adaptation results in an increase of activation which brings the neural populations in $u_{A C T}$ closer to threshold and thus reduces the time to action onset. This can be seen when comparing again the time courses of activation in a new recall trial, 7(b). Table I compares the delays before and after the application of the adaptation dynamics. It shows that the delay can be drastically reduced in a single trial (for a discussion of the adaptation rate parameter $\beta$ see [22]).

\section{DISCUSSION}

In the present study, we have presented first results of our ongoing research on endowing autonomous robots with an adaptive action timing capacity. The neurodynamics approach takes inspiration from neural processing mechanism that are believed to support time-dependent cognition in humans and other animals. The human capacity to learn temporal regularities in the environment and to continuously adapt to changing temporal conditions is not well described by a simple clocklike mechanism [10], [12]. Here we have shown that the robot can successfully adapt the learned timing pattern of an action sequence to the occurrence of predictable sensory cues without relying on a computer clock. Towards our ultimate goal of natural human-robot interactions, we are currently testing the timing model as part of a DNF control architecture for joint action [2] in object transfer tasks [14]. The synchronization cue is here the hand of the user at the exchange position. We believe that a human-like temporal cognition capacity for robots that integrates the impact of stimulus properties, context and attention on time perception [12] will make the robot more

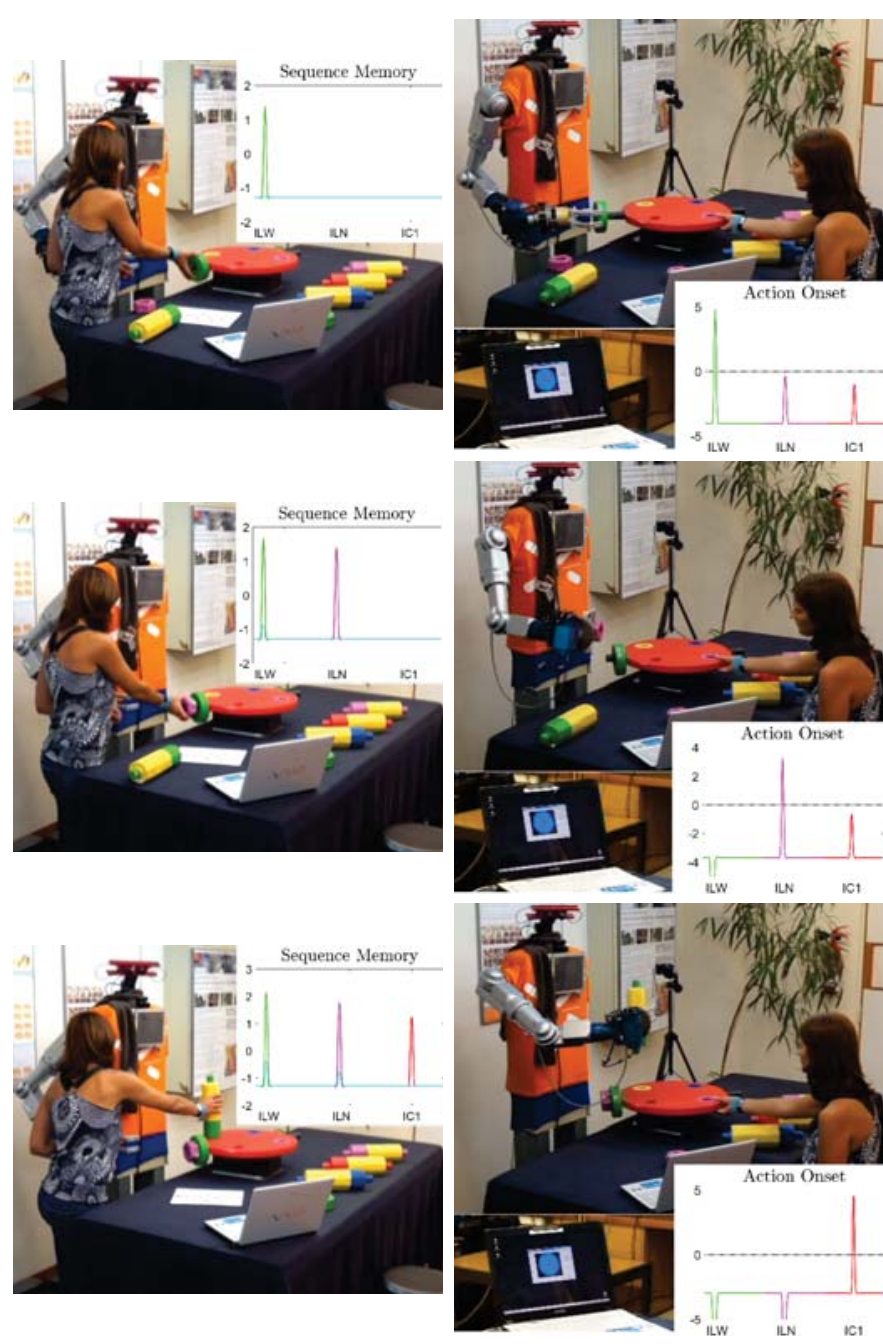

Fig. 4: Video snapshots of the sequence learning (left column) and sequence execution (right column).

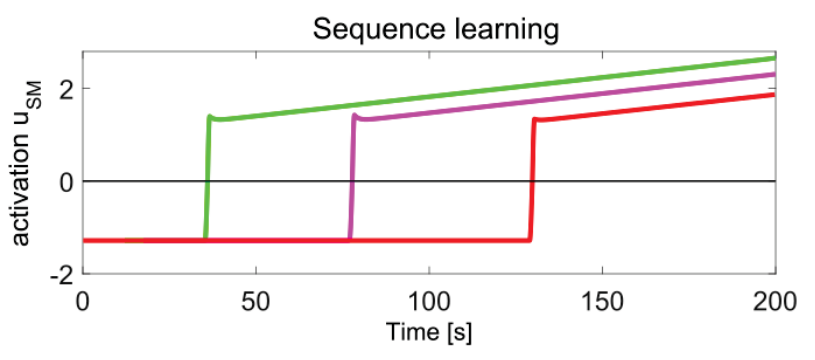

Fig. 5: Time course of population activity in $u_{S M}$ during sequence demonstration. 


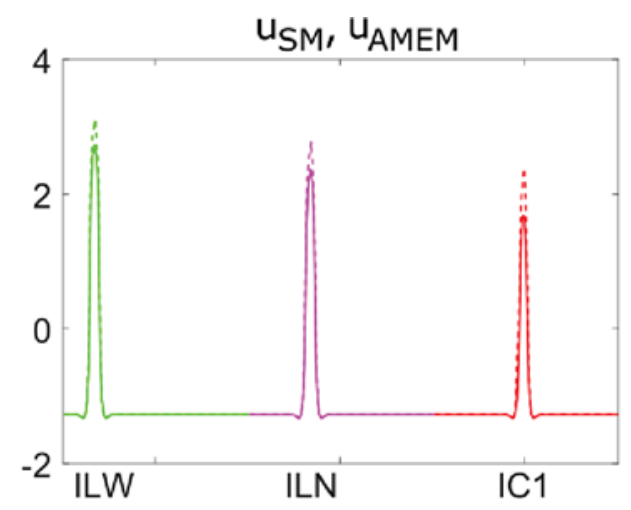

Fig. 6: Local adaptation of resting level in $u_{A M E M}$. The activation patterns in the Perceptual Sequence Memory field $u_{S M}$ (solid line) and the Action Onset Memory field $u_{A M E M}$ (dotted line) are compared.

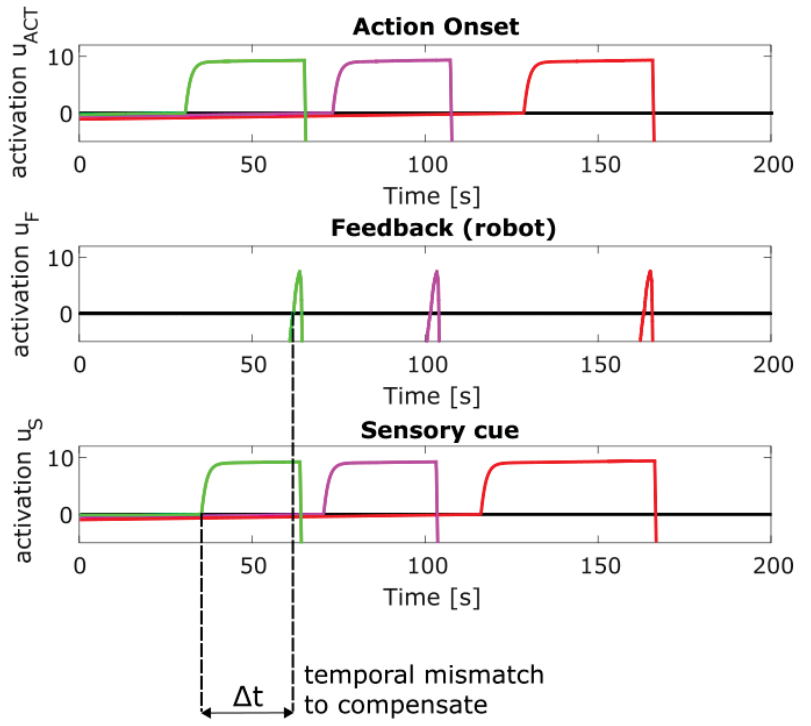

(a) First execution trial
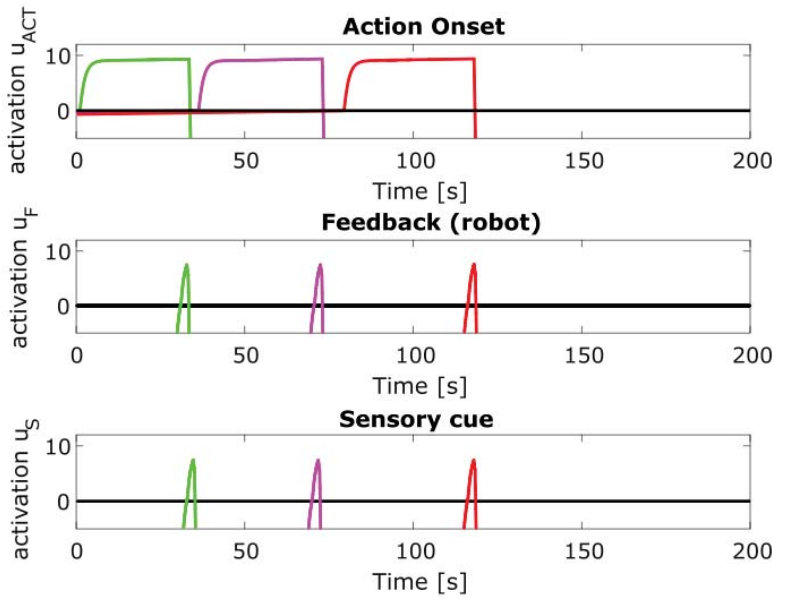

(b) Second execution trial

Fig. 7: Comparison of time courses of population activity in $u_{A C T}, u_{F}$ and $u_{S}$ during the first (a) and second (b) execution trial. predictable for the human user. This in turn will increase user acceptance.

In line with recent neurophysiological findings, the DNF model builds on the assumption that neural population involved in higher brain functions such as memory, planning and decision making are themselves time keeper. It is thought that the firing rate dynamics of persistent activity allows the neurons to measure the passage of time [10]. The neurodynamics approach differs fundamentally from implementations of robot time perception based on classical artificial intelligence (AI) methods (for discussion see [3], [5], [11],[17]). They typically assume that time is an independent behavioral dimension that can be directly obtained by a computer clock for labeling events. For instance, in [11] a Bayesian approach is applied in a human-robot assembly task to predict the duration of assembly steps based on explicit time measurements and the prior that individual assembly step have approximately equal durations. Changing the temporal order of task execution or the duration of individual steps would require to coordinate the separate timing module with other cognitive modules representing working memory and decision planning. In the DNF model, timed behavior emerges from the interaction of neural representation of sensory and motor events with the constraint that the slope of the buildup of population activity in the memory and the recall fields are correlated (not necessarily a linear dynamics). Very recent neurophysiological findings seem to support this assumption (for discussion see [10]). Ramping activity as a neural integrator of time is however not the only neural mechanism which is believed to support time-dependent cognition. In a distinct but conceptually related model class known as population clocks, the evolution of the collective state space of recurrently connected neurons spanned by the firing rates is used to represent the passage of time [4]. The synaptic weights are first learned to elicit a trajectory through state space in a robust and reproducible manner. Output units are then trained to recognize a specific pattern which serves as a readout of time. The neural population trajectory in the DNF model is shaped by the spatial interactions supporting persistent activity which we assume to be already in place [20]. The model thus supports activation-based learning which is commonly believed to be much faster than weight-based learning [19]. The DNF framework is not only compatible with timing models based on ramping activity but also with accounts based on neural oscillators. A coupled system of two field equations of Amari-type describing an activator-inhibitor system can be used to model stable, reproducible oscillations [18]. Measuring time is linked to counting the number of oscillatory cycles. Integrating such timing mechanism could be beneficial in application in which the bump height is used to represent additional input information like for instance saliency or probability.

In the present experiment, several aspects have been simplified to focus on the timing capacity. This explains to some extent the rapid learning and adaptation of the robot in just two execution trials. First, we assume that the perceptual memory of the demonstrated assembly sequence is correct. In [9] we have 
discussed a model extension that allows to iteratively correct initial encoding errors. Second, the use of a color signal as a synchronization cue obviously simplifies the synchronization challenge since its occurrence can be detected easily and with high temporal precision. In joint action, even the duration of globally predictable actions of the co-worker will vary from trial to trial. With the present model implementation, the robot would adapt to the memorized pattern from the last trial to timely initiate its complementary behavior in the current joint action. This is a reasonable strategy if temporal precision of action execution is relatively high. It also ensures that the robot may rapidly adapt to other users which may show different temporal patterns.

Given the fundamental importance that the processing of time plays for our everyday activities, assistive robots should be endowed with a temporal cognition capacity to be more easily accepted by human users [14]. We believe that neurodynamics offers key processing principles that may guide the development of a new generation of cognitive robots.

\section{ACKNOWLEDGMENT}

This research was supported by the Marie Curie Network for Initial Training NETT, FCT through the $\mathrm{PhD}$ fellowship PD/BD/128183/2016, the FCT-Research Center CMAT (PEstOE/MAT/UI0013/2014), and FCT - Algoritmi research Centre (COMPETE: POCI-01-0145-FEDER-007043 and FCT Fundação para a Ciência e Tecnologia within the Project Scope: UID/CEC/00319/2013).

\section{REFERENCES}

[1] S. Amari. Dynamics of pattern formation in lateral-inhibition type neural fields. Biological Cybernetics, 27(2):77-87, 1977.

[2] E. Bicho, W. Erlhagen, L. Louro, and E. Costa e Silva. Neurocognitive mechanisms of decision making in joint action: A human-robot interaction study. Human Movement Science, 30(5):846 - 868, 2011.

[3] F. Broz, I. R. Nourbakhsh, and R. G. Simmons. Planning for humanrobot interaction using time-state aggregated pomdps. In $A A A I$, volume 8, pages 1339-1344, 2008.

[4] D. V. Buonomano and R. Laje. Population clocks: motor timing with neural dynamics. Trends in cognitive sciences, 14(12):520-527, 2010.

[5] C. Chao and A. L. Thomaz. Timing in multimodal turn-taking interactions: Control and analysis using timed petri nets. Journal of HumanRobot Interaction, 1(1), 2012.
[6] S. Coombes and M. Owen. Exotic dynamics in a firing rate model of neural tissue with threshold accommodation. AMS Contemporary Mathematics, 44:123-144, February 2007.

[7] W. Erlhagen and E. Bicho. The dynamic neural field approach to cognitive robotics. Journal of Neural Engineering, 3:36-54, 2006.

[8] F. Ferreira, W. Erlhagen, and E. Bicho. Multi-bump solutions in a neural field model with external inputs. Physica D: Nonlinear Phenomena, 326:32-51, 2016.

[9] F. Ferreira, W. Erlhagen, E. Sousa, L. Louro, and E. Bicho. Learning a musical sequence by observation: A robotics implementation of a dynamic neural field model. In Development and Learning and Epigenetic Robotics (ICDL-Epirob), 2014 Joint IEEE International Conferences on, pages $157-162,2014$.

[10] G. T. Finnerty, M. N. Shadlen, M. Jazayeri, A. C. Nobre, and D. V. Buonomano. Time in cortical circuits. Journal of Neuroscience, 35(41):13912-13916, 2015 .

[11] S. Glasauer, M. Huber, P. Basili, A. Knoll, and T. Brandt. Interacting in time and space: Investigating human-human and human-robot joint action. In RO-MAN, 2010 IEEE, pages 252-257. IEEE, 2010.

[12] S. Grondin. Timing and time perception: a review of recent behavioral and neuroscience findings and theoretical directions. Attention, Perception, \& Psychophysics, 72(3):561-582, 2010.

[13] J. Hass and D. Durstewitz. Time at the center, or time at the side? Assessing current models of time perception. Current Opinion in Behavioral Sciences, 8:238 - 244, 2016.

[14] A. Koene, M. P. Remazeilles, M. Prada, A. Garzo, M. Puerto, S. Endo, and A. M. Wing. Relative importance of spatial and temporal precision for user satisfaction in human-robot object handover interactions. Proc. New Frontiers in Human-Robot Interaction, 14, 2014.

[15] J. L. Krichmar, J. Conradt, and M. Asada. Neurobiologically inspired robotics: Enhanced autonomy through neuromorphic cognition. Neural Networks, 72:1 - 2, 2015. Neurobiologically Inspired Robotics: Enhanced Autonomy through Neuromorphic Cognition.

[16] P. A. Lewis and R. C. Miall. Remembering the time: a continuous clock. Trends in cognitive sciences, 10(9):401-406, 2006.

[17] M. Maniadakis and P. Trahanias. Temporal cognition: a key ingredient of intelligent systems. Frontiers in neurorobotics, 5:2, 2011.

[18] G. Schöner. Timing, clocks, and dynamical systems. Brain and cognition, 48(1):31-51, 2002.

[19] E. Sousa, W. Erlhagen, F. Ferreira, and E. Bicho. Off-line simulation inspires insight: a neurodynamics approach to efficient robot task learning. Neural Networks, 72:123-139, 2015.

[20] X. J. Wang. Synaptic reverberation underlying mnemonic persistent activity. Trends in neurosciences, 24(8):455-463, 2001.

[21] C. Wardak, S. Ramanol, O. Guipponi, P. Boulinguez, and S. B. Ben Hamed. Proactive inhibitory control varies with task context. European Journal of Neuroscience, 36(11):3568-3579, 2012.

[22] W. Wojtak, F. Ferreira, W. Erlhagen, and E. Bicho. Learning joint representations for order and timing of perceptual-motor sequences: a dynamic neural field approach. In Neural Networks (IJCNN), 2015 International Joint Conference on, pages 3082-3088. IEEE, 2015. 